\title{
A First Principles Estimate of Finite Size Effects in Quark-Gluon Plasma Formation
}

\author{
Andy Gopie and Michael C. Ogilvie \\ Department of Physics, Washington University, St. Louis, MO 63130
}

November 10, 2018

\begin{abstract}
Using lattice simulations of quenched QCD we estimate the finite size effects present when a gluon plasma equilibrates in a slab geometry, i.e., finite width but large transverse dimensions. Significant differences are observed in the free energy density for the slab when compared with bulk behavior. A small shift in the critical temperature is also seen. The free energy required to liberate heavy quarks relative to bulk is measured using Polyakov loops; the additional free energy required is on the order of $30-40 \mathrm{MeV}$ at $2-3 T_{c}$.

PACS numbers: 11.10.Wx 12.38.Gc 12.38.Mh
\end{abstract}

\section{INTRODUCTION}

The formation of a quark-gluon plasma in a central heavy ion collision is generally assumed to take place in a coin-shaped region roughly 1 fermi in width, with radius comparable to the radii of the colliding nuclei, which is to say several fermi. While lattice gauge theory has given us information about bulk thermodynamic behavior, finite size effects have up to now been studied using simplified, phenomenological models. In this work, we study via lattice gauge theory simulations the behavior of a gluon plasma restricted to a slab geometry, with the longitudinal width much smaller than the transverse directions. This inner region is heated to temperatures above the bulk deconfinement temperature, surrounded by an outer region which is kept at a temperature below the deconfinement temperature. This outer region provides confining boundary conditions for the inner region. From our simulations, we derive information about the thermodynamics of the gluon plasma in a slab geometry, the effective non-equilibrium surface tension and the free energy cost of creating quarks relative to bulk.

Measurements of the equilibrium surface tension $\alpha_{0}$ of pure $\mathrm{SU}(3)$ lattice gauge theory (quenched lattice QCD) show that the dimensionless ratio $\alpha_{0} / T_{c}^{3}$ is small. For the case of an $N_{s}^{3} \times 4$ lattice, $\alpha_{0} / T_{c}^{3} \approx 0.0292(22)$. [1] A simple estimate of surface tension effects on the transition can be obtained from a simplified model in which only volume and surface terms appear, as in the bag model. [2] Since we are interested in the pure gauge theory, we take the free energy density of the hadronic phase to be zero, neglecting the contribution of glueballs, whose masses are large compared to the deconfinement temperature. The free energy of a gluon plasma of volume $V$ and surface area $A$ we take to be given by

$$
F=\left(B-18 \frac{\pi^{2}}{90} T^{4}\right) V+\alpha_{0} A
$$

The bag constant $B$, which here is simply related to the latent heat at the deconfinement transition, is taken to be $B=(200 M e V)^{4}$. Taking $\alpha_{0} / T_{c}^{3}$ as above, the bulk critical temperature to be $T_{c}=260 \mathrm{MeV}$ and the slab width as $w=1 \mathrm{fm}$. leads to an increase in $T_{c}$ of about $3 \%$. Naively, finite size effects due to the surface tension should be small.

Other contributions to finite size effects come from a variety of sources. In the case of systems with nonabelian symmetries, global color invariance produces an additional finite volume effect which will not be considered here. [3] [4] [5] In general, finite size effects lead to a rounding of the transition. [6] This can be taken into account in the bag model by a Maxwell construction, leading to mixed phases and a broadened critical region. A recent treatment for the quark gluon plasma can be found in [7]. We have attempted to avoid these finite volume effects by making the transverse dimensions large.

\section{METHODOLOGY}

In lattice calculations, finite temperature is introduced by the choice of $N_{t}$, the extent of the lattice in the (Euclidean) temporal direction. The relation of physical temperature $T$ to $N_{t}$ and the lattice spacing $a$ is simply $T=1 / N_{t} a$. The lattice spacing $a$ implicitly depends on the gauge coupling $\beta$ in a way determined by the renormalization group equations. To lowest order in perturbation theory, the relation is given by

$$
a \Lambda_{L}=\left(\frac{\beta}{2 N b_{0}}\right)^{b_{1} / 2 b_{0}^{2}} \exp \left[-\beta / 4 N b_{0}\right]
$$


where $\Lambda_{L}$ is renormalization group invariant and the renormalization group coefficients $b_{0}$ and $b_{1}$ are given by

$$
b_{0}=\frac{11 N}{48 \pi^{2}} \quad, \quad b_{1}=\frac{34}{3}\left(\frac{N}{16 \pi^{2}}\right)^{2}
$$

In analyzing our data, we used the renormalization group results given in reference [8], which are determined directly from lattice simulations, and contain non-perturbative information about the renormalization group flow.

By allowing the coupling constant $\beta=6 / g^{2}$ to vary with spatial location, a spatially dependent temperature can be introduced. We have chosen the temperature interface to be sharp, in such a way that the lattice is divided into two spatial regions, one hotter and one colder. Figure 1 shows the slab geometry that was used for simulations. By varying the spatial and temporal plaquette couplings independently, it is possible in principle to make the spatial lattice spacing constant in physical units, at least in a slab geometry. [9] However, the quenched approximation simplifies the role of the cold region. In the quenched approximation below $T_{c}$, the dominant excitation at low energies is the scalar glueball. Temperatures near $T_{c}$ are smaller than glueball masses by about a factor of four, so glueballs play no essential role in the thermodynamics, and the pressure in the hadronic phase is essentially zero. For this reason, we expect the slab thermodynamics to be largely insensitive to the precise temperature of the region outside the slab, as long as it is sufficiently low. The outer region thus merely provides boundary conditions for the slab. A numerical check of this assumption is discussed below. In full QCD, this insensitivity to the outer temperature would not hold, due to pions. Note that the role of boundary conditions here is quite different from those relevant for the QCD phase transition in the early universe. Assuming the transition in full QCD is first order, nucleation will occur as the universe cools below the transition. If the equilibrium surface tension is small, the nucleation rate will be high, and no significant undercooling is expected. In this case, a spherical geometry is relevant, and simulations have been performed where $\beta_{\text {in }}$ and $\beta_{\text {out }}$ have been taken to be near $T_{c}$. [10], [11] Note that such simulations are not directly relevant to heavy ion collisions, since substantial temperature gradients will develop by the time the expanding quark-gluon plasma becomes approximately spherical.

Another difference between this system and nearequilibrium systems lies in fluctuations of the interface. For equilibrium or near-equilibrium systems, interfacial fluctuations play a significant role. In three dimensions, we expect that an equilibrium planar interface would always be in the rough phase. Interfacial fluctuations associated with roughening lead to a universal subleading correction to the planar interfacial free energy. [12], 13] In the case of nucleation, interfacial fluctuations play an important role in the prefactor of the nucleation rate via the functional determinant. [14] In the model we are considering, the interface is pinned by the sharp temperature interface, which may drastically reduce fluctuations. It seems likely to us that this is a physical effect. Furthermore, the surface modes associated with roughening are long-wavelength modes which are likely to require substantial time to equilibrate. We conclude that interfacial fluctuations are unlikely to play the same role in an an expanding quark-gluon plasma created by a heavy ion collision that they would in an equilibrium situation.

In our simulations, we work with slabs of fixed lattice width, $w=6 a$, rather than of fixed physical width. Since $N_{t}=4, w T$ is fixed at $3 / 2$. Maintaining a fixed width in unphysical lattice units means that the width of the slab in physical units varies by almost a factor of 3 over the range of $\beta$ values used, from approximately 1.2 fermi to 0.5 fermi. At higher temperatures, this is somewhat smaller than the longitudinal size of the plasma formation region expected in heavy ion collisions.

The use of equilibrium statistical mechanics to study gluon plasma properties during the early stages of plasma formation may appear suspect. It is useful to compare the conditions here with those of the Bjorken model. 15. In this model, the temperature is specified on an initial surface of constant proper time $\tau_{0}$, and has a simple scaling behavior given by

$$
T(\tau)=T_{0}\left(\tau_{0} / \tau\right)^{c_{s}^{2}}
$$

where $c_{s}$ is the speed of sound. For an ideal gas, $c_{s}=$ $\sqrt{1 / 3}$. This can be used to estimate the spatial variation of the temperature of a quark-gluon plasma shortly after formation in a central collision. For example, when a coin-shaped region of width 1 fermi has expanded to 1.5 fermi, the variation in temperature is only from $0.8 T_{0}$ at the center of the coin to $T_{0}$ at its edges. It has been argued that $c_{s}$ may be much smaller than $\sqrt{1 / 3}$ near $T_{c}$ [16], [17], which would further decrease variation in $T$. Thus the assumption of constant temperature is likely to be a good approximation to the actual early stages of a quark-gluon plasma formed in a heavy ion collision. Although, a non-trivial temperature profile could be incorporated by adjusting the spatial dependence of the coupling constants, it is neither necessary nor desirable. A more significant difference between the conditions studied here and those of the Bjorken model lies in the velocity distribution. The Bjorken model leads to a space-time dependent velocity distribution whereas lattice models are restricted to an average velocity of zero. Adding even a constant average velocity has a technical difficulty similar to the well-known problem of adding a non-zero chemical potential to lattice models.

The free energy density $f$ for the slab was obtained using the standard method [18] of integrating the lattice action with respect to $\beta$. We use a convenient convention for the sign of $f$ that is opposite the usual one. In the bulk case, $f$ is then identical to the pressure $p$. 


$$
\left.\frac{f}{T^{4}}\right|_{\beta_{\text {out }}} ^{\beta}=N_{t}^{4} \int_{\beta_{\text {out }}}^{\beta} d \beta^{\prime}\left[\langle S\rangle_{T}-\langle S\rangle_{0}\right]
$$

where $\langle S\rangle$ is the expectation value of a plaquette averaged over the region of interest:

$$
S=\frac{1}{N} \operatorname{Re} \operatorname{Tr} U_{p}
$$

The subscripts $T$ and 0 denote expectation values measured at finite and zero temperature, respectively. As in the bulk case, it is necessary to subtract the zerotemperature action density expectation value from the finite temperature expectation value, removing terms which would give divergences in the continuum limit. The subtracted term is obtained from a zero temperature simulation with the same pair of $\beta$ values. This is clearly necessary because the introduction of two regions with differing $\beta$ values introduces an interface even at zero temperature. However, there is the possibility that new divergences are introduced by the interface between the two regions. Such divergences would manifest in the need for counterterms on the boundary between the two regions. In general, quantum field theories with boundaries develop divergences that are not present in infinite volume or with periodic boundary conditions. Symanzik 119 has shown to all orders in perturbation theory that in the case of $\phi^{4}$ with so-called Schrodinger functional boundary conditions, the theory is finite in perturbation theory after adding all possible boundary counterterms of dimension $d \leq 3$ consistent with the symmetries of the theory. It is generally believed that this result applies as well to all renormalizable field theories and general boundary conditions, but a proof is lacking. Luscher et al. [20] have shown for gauge theories that at one loop no new divergences are introduced by Schrodinger functional boundary conditions. This is consistent with the non-existence of gauge-invariant local fields of dimension $\leq 3$ in pure Yang-Mills theory.

In order to take advantage of the data on bulk thermodynamics provided by the Bielefeld group [8], we worked consistently with lattices of overall size $16^{3} \times 4$. Up to 8000 initialization sweeps were used, and up to 30,000 sweeps were used for measurements. Measurements were made every 10 sweeps, and corrected for autocorrelation. The values used for each subtraction come from $16^{4}$ lattices with identical values of $\beta_{\text {in }}$ and $\beta_{\text {out }}$. The value of $\beta_{\text {out }}$ was held fixed at 5.6 while $\beta_{\text {in }}$ varied from 5.6 to 6.3. For comparison, the bulk transition for $N_{t}=4$ occurs at $\beta_{c}\left(N_{t}=4, N_{s}=16\right)=5.6908(2)$ $\beta_{c}\left(N_{t}=4, N_{s}=\infty\right)=5.6925(5)$. [8]

\section{FREE ENERGY OF GLUONS}

Figure 2 shows the free energy density $f / T^{4}$ versus $T / T_{c}$ compared with the bulk pressure. The free energy in the slab is lower than the bulk value by almost a factor of two at $2 T_{c}$. It appears that the slab value is slowly approaching the bulk value, but other behaviors are also possible. Calculations of the finite-temperature contribution to the Casimir effect for a free Bose field contained between two plates show that $f / T^{4}$ has a nontrivial dependence on the dimensionless combination $w T$. [21], 22] It is natural to ask if the corrections to the free energy seen here can be accounted for by the conventional Casimir effect. A straigtforward calculation of the free energy of a non-interacting gluon gas confined to a slab shows an increase in the free energy density over the bulk value by a factor of about 1.63 at $w T=3 / 2$. The Casimir effect alone is thus unable to explain the reduction of the free energy observed in our simulations. We are currently investigating more elaborate theoretical models which include the effect of a non-trivial Polyakov loop.

A consistency check was performed on the surface effects using a method originally developed for measuring the equilibrium surface tension. 23] The free energy density was calculated for a system at $\beta_{\text {in }}=6.0$ by performing simulations with $\beta_{\text {in }}$ fixed at 6.0 and $\beta_{\text {out }}$ varying from 5.6 to 6.0. Combining these results with the bulk data of reference [8] creates a path equivalent to varying $\beta_{\text {in }}$ while holding $\beta_{\text {out }}$ fixed. Figure 3 shows the two equivalent paths. For $\beta_{\text {in }}=6.0$ and $\beta_{\text {out }}=5.6$, this gives $f / T^{4}=0.65 \pm 0.04$, to be compared with $f / T^{4}=0.69 \pm 0.03$ for the direct calculation.

The major source of systematic error lies with the choice of boundary conditions for the slab, here set by $\beta_{\text {out }}$. We have estimated the effects of varying $\beta_{\text {out }}$ by performing simulations at $\beta_{\text {in }}=6.2$ and $\beta_{\text {out }}=5.5$ on $16^{3} \times 4$ and $16^{4}$ lattices. These results suggest that lowering $\beta_{\text {out }}$ from 5.6 to 5.5 reduces the free energy by roughly 10 percent at $\beta_{\text {in }}=6.2$.

\section{SURFACE TENSION}

The equilibrium surface tension $\alpha_{0}$ can be defined from the excess free energy as $\beta_{\text {in }}$ and $\beta_{\text {out }}$ approach $\beta_{c}$, the bulk critical value, with the width $w$ simultaneously taken to infinity. It is common to assume that this quantity can also be used to characterize non-equilibrium conditions as well. As we have seen in the introduction, the small equilibrium value of $\alpha_{0} / T^{3}$ would lead to negligible surface effects in QCD.

We define an effective surface tension $\alpha(w, T)$ by

$$
f=p-2 \alpha(w, T) / w
$$

where the notation $\alpha(w, T)$ recognizes that the surface tension $\alpha$ does depend on the width of the slab and the internal and external temperatures. The factor of 2 occurs because the slab has two faces. In the limits where $T$ approaches $T_{c}$ and $w$ goes to infinity, this quantity approaches $\alpha_{0}$. Figure 4 shows $\alpha(w, T) / T^{3}$ versus $T / T_{c}$ for $w T=3 / 2$; representative error bars are shown. 
The value at $\beta=5.7,0.056 \pm 0.002$ is substantially higher than the value $\alpha_{0}=0.0292 \pm 0.0022$ given in reference [1]. We attribute this to two effects: in our case $\beta_{\text {out }}$ is fixed at 5.6 , whereas for equilibrium measurements it is extrapolated to $\beta_{c}$, and our finite value of the width $w$ also acts to increase $\alpha(w, T)$ over the equilibrium value as measured in simulations at large $w$. Away from the bulk critical point, $\alpha / T^{3}$ rises quickly to a peak at about $1.4 T_{c}$, and then falls slowly as $T$ increases. A large non-equilibrium surface tension has also been observed in measurements of the equilibrium surface tension, where these effects were obstacles to obtaining $\alpha_{0}$. 23]

\section{FREE ENERGY OF QUARKS}

The Polyakov loop defined by

$$
P(\vec{x})=\left(1 / N_{c}\right) \operatorname{Tr} \mathcal{P} \exp \left[i \int_{0}^{1 / T} A_{0}(\vec{x}, \tau) d \tau\right]
$$

is the order parameter for the deconfinement transition in pure (quenched) gauge theories. In the case of $S U(N)$, there is a global $Z(N)$ symmetry which ensures at low temperature that the expectation value $\langle\operatorname{Tr} P\rangle$ is 0 . At sufficiently high temperatures, this symmetry is spontaneously broken. The expectation value of the Polyakov loop can also be interpreted in terms of the free energy of an isolated, infinitely heavy quark $F_{Q}$ :

$$
\langle P(\vec{x})\rangle=\exp \left[-F_{Q}(\vec{x}) / T\right]
$$

In the low-temperature confined phase, $F_{Q}$ is taken to be infinite, whereas in the high temperature phase it is finite.

Direct extraction of $F_{Q}$ from computer simulations is problematic, because the expectation value has a multiplicative, $\beta$-dependent ultraviolet divergence. This divergence can be eliminated when comparing bulk expectation values to those in finite geometries. We define

$$
\Delta F_{Q}(\vec{x})=-T \ln \left[P_{\text {slab }}(\vec{x}) / P_{\text {bulk }}\right]
$$

as the excess free energy required to liberate a heavy quark in the slab geometry relative to bulk quark matter at the same temperature. This technique can also be used in, e.g., a spherical geometry, which is relevant for nucleation. [24], 10]

Unlike the thermodynamics, the Polyakov loop profile is expected to be sensitive to the outer temperature, since the Polyakov loop will decay away from the interface as $\exp \left[-\sigma r / T_{\text {out }}\right]$, where $\sigma$ is the string tension. In figure 5, we show the expectation value for the Polyakov loop versus $\mathrm{z}$ measured in lattice units for several values of $\beta$. Each curve is normalized by dividing the values of the Polyakov loop by the bulk expectation value at the corresponding value of $\beta$. Error bars are shown only for even values of $\mathrm{z}$. It is clear that a significant change occurs between $\beta=5.8 \quad\left(T=1.23 T_{c}\right)$ and $\beta=5.85\left(T=1.36 T_{c}\right)$. For larger values of $\beta, \Delta F_{Q}$ diminishes to a value of approximately $30-40 \mathrm{MeV}$ in the middle of the slab. In table one, we list $\beta, T$, slab width in fermis, width of the core in fermis, and $\Delta F_{Q}$ in $\mathrm{MeV}$ for representative values. The width of the core is calculated by interpolating the Polyakov loop profiles and determining the region where the slab expectation value is greater than $80 \%$ of the bulk value. All conversions to physical units are performed by taking the string tension $\sigma$ to be $(425 \mathrm{MeV})^{2}$, which implies $T_{c}=254 \mathrm{MeV}$. [8]

\section{CONCLUSIONS}

There are significant deviations in the slab geometry from bulk behavior and the ideal gas law, arising from a strong non-equilibrium surface-tension. This nonequilibrium surface tension can be an order of magnitude greater than the equilibrium value. Surface tension effects also produce a mild elevation of the apparent critical temperature. Measurement of Polyakov loop expectation values relative to bulk shows that the suppression of heavy quark production due to the slab geometry is small.

There is little doubt that most of the effects seen can be attributed to the smallness of the width $w$ relative to the natural interfacial width. If $w$ were made arbitrarily large, the two interfaces on either side of the slab would be independent, and each would have some thickness $t$, as measured, say, from the behavior of the Polyakov loop. In this situation, the thin wall approximation, familiar in nucleation theory, is valid, and the free energy can be decomposed into volume and surface terms. [25] As $w$ is reduced and becomes commensurate with $t$, the thin wall approximation breaks down. Intuitively, it becomes impossible to fit two interfaces plus a bulk region between them into the width available. For any fixed temperature and sufficiently small $w$, no true bulk phase can be formed. This is a physical concern for quark-gluon plasma formation in heavy ion collisions, independent of any computer simulation or model.

There are good reasons to call our result an estimate rather than a calculation. Although lattice gauge theory simulations of bulk behavior can be made arbitrarily accurate in principle, in this case there is some uncertainty in the precise exterior boundary conditions appropriate, and indeed in the applicability of equilibrium thermodynamics at this early stage of quark-gluon plasma formation. However, this seems like the best estimate available now, and further refinements are possible. An alternative approach might be based on real-time simulations; while this area is the subject of active research, it is still in its infancy. [26], 27]

We have not yet explored the nature of the phase transition, which will require some care. One interesting possibility is that the order of the transition might change as 
the width changes. The deconfinement transition in bulk quenched finite temperature QCD is in the universality class of the three-dimensional three-state Potts model, which has a first-order phase transition. As the width of the slab becomes commensurate with the correlation length near $T_{c}$, the phase transition should cross over to the universality class of the two-dimensional three-state Potts model. The two-dimensional three-state Potts model has a second-order phase transition, so it is possible that the order of the transition may change. [28] The correlation length at the bulk transition is known to be large [29], so it is likely that the transverse correlation length in the gluonic sector is much larger in the slab geometry than in bulk, even if crossover does not take place.

\section{ACKNOWLEDGEMENTS}

We wish to thank the U.S. Department of Energy for financial support under grant number DE-FG02-91ER40628.

[1] Y. Iwasaki, K. Kanaya, Leo Kärkkäinen, K. Rummukainen and T. Yoshie, Phys.Rev. D49 , 3540 (1994).

[2] J. Cleymans, R. Gavai and E. Suhonen, Phys. Rep. 130, 217 (1986).

[3] K. Redlich and L. Turko, Z. Phys. C5, 201 (1980).

[4] L. Turko, Phys. Lett. B104, 153 (1981).

[5] B.S. Skagerstam, Phys. Lett. B133, 419 (1983).

[6] K. Binder and D.P. Landau, Phys. Rev. B30, 1477 (1984).

[7] C. Spieles, H. Stöcker, and C. Greiner, Phys. Rev. C57, 908 (1998).

[8] G. Boyd, J. Engels, F. Karsch, E. Laermann, C. Legeland, M. Lutgemeier and B. Petersson, Nucl. Phys. B469, 419 (1996).

[9] G. Burgers, F. Karsch, A. Nakamura and I.O. Stamatescu Nucl. Phys. B304 587 (1988).

[10] K. Kajantie, L. Kärkkäinen and K. Rummukainen, Phys. Lett. 286B , 125 (1992).

[11] S. Huang, J. Potvin and C. Rebbi, Int. J. Mod. Phys. C3 , 931 (1992).

[12] M. Luscher, K. Symanzik and P. Weisz, Nucl.Phys. B173, 365 (1980).

[13] M. Luscher, Nucl.Phys. B180, 317 (1981).

[14] J. Langer, Ann. Phys. 41, 108 (1967).

[15] J. Bjorken, Phys. Rev. D49, 140 (1983).

[16] C. Schmid, D. Schwarz and P. Widerin, Phys. Rev. Lett. 78. 5468 (1997).

[17] K. Jedamzik, Phys. Rev. D55, 5871 (1997).

[18] J. Engels, J. Fingberg, F. Karsch, D. Miller and M. Weber, Phys. Lett. B252, 625 (1990).

[19] K. Symanzik in Mathematical Problems in Theoretical Physics, R. Schrader et al., eds., Spinger, New York (1982).
[20] M. Luscher, R. Narayanan, P. Weisz and U. Wolff, Nucl. Phys. B384, 168 (1992).

[21] J. Mehra, Physica 37, 145 (1967).

[22] G. Plunien, B. Müller and W. Greiner, Phys. Rep. 134, 87 (1986).

[23] S. Huang, J. Potvin, C. Rebbi and S. Sanielevici, Phys. Rev. D42, 2864 (1990); Erratum ibid. D43, 2056 (1991).

[24] M. Ogilvie, Nucl. Phys. B (Proc. Suppl.) 30, 354 (1993).

[25] S. Coleman, Phys. Rev. D15, 2929 (1977); C. Callan and S. Coleman, Phys. Rev. D16, 1762 (1977).

[26] E. Iancu, hep-ph/9807299.

[27] S.A. Bass, B. Muller an, W. Poschl, nucl-th/9808011; W. Poschl and B. Muller, nucl-th/9808031.

[28] Y. Shnidman and E. Domany, J. Phys. C14, L773 (1981).

[29] M. Fukugita, M. Okawa and A. Ukawa, Nucl. Phys. B337, 181 (1990).

TABLE I. $\beta, T$, slab width in fermis, width of the core in fermis, and $\Delta F_{Q}$ in $\mathrm{MeV}$ for representative values.

\begin{tabular}{lllll}
\hline \hline$\beta$ & $T(\mathrm{MeV})$ & $\mathrm{w}(\mathrm{fm})$. & $w_{\text {core }}(\mathrm{fm})$. & $\Delta F_{Q}(\mathrm{MeV})$ \\
\hline 5.8 & 313 & 0.95 & 0 & 169 \\
5.85 & 346 & 0.86 & 0.40 & 57 \\
6.0 & 455 & 0.65 & 0.48 & 41 \\
6.2 & 624 & 0.47 & 0.40 & 31 \\
\hline \hline
\end{tabular}


FIG. 1. Schematic Drawing of slab geometry.

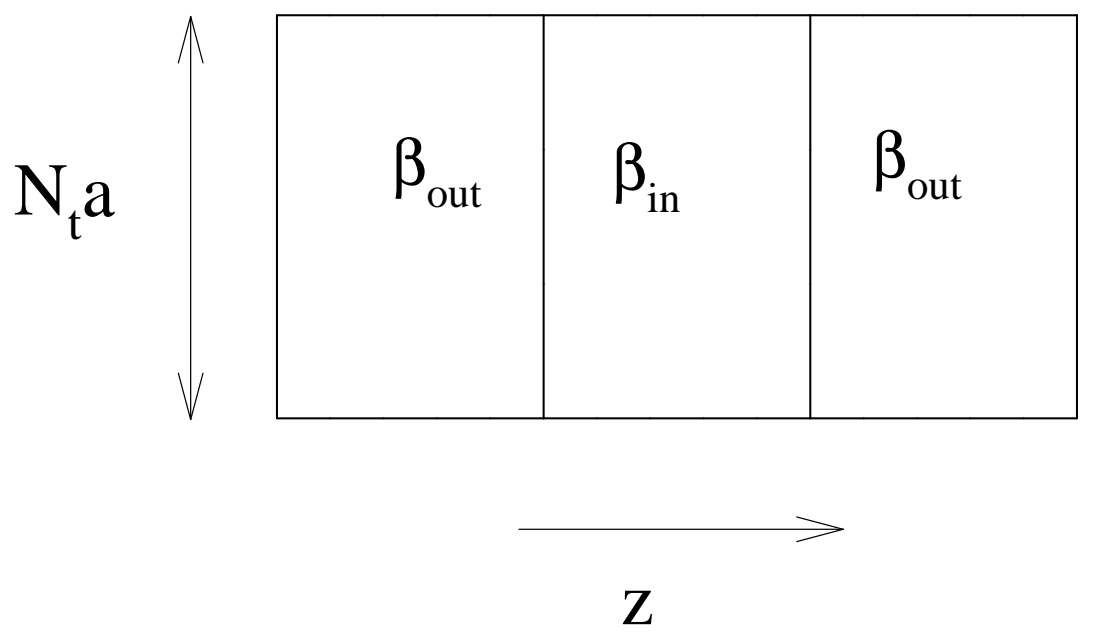


FIG. 2. Free energy density $f / T^{4}$ versus $T / T_{c}$ for the slab geometry at $w T=3 / 2$ compared with bulk behavior. The bulk data is from reference 8 .

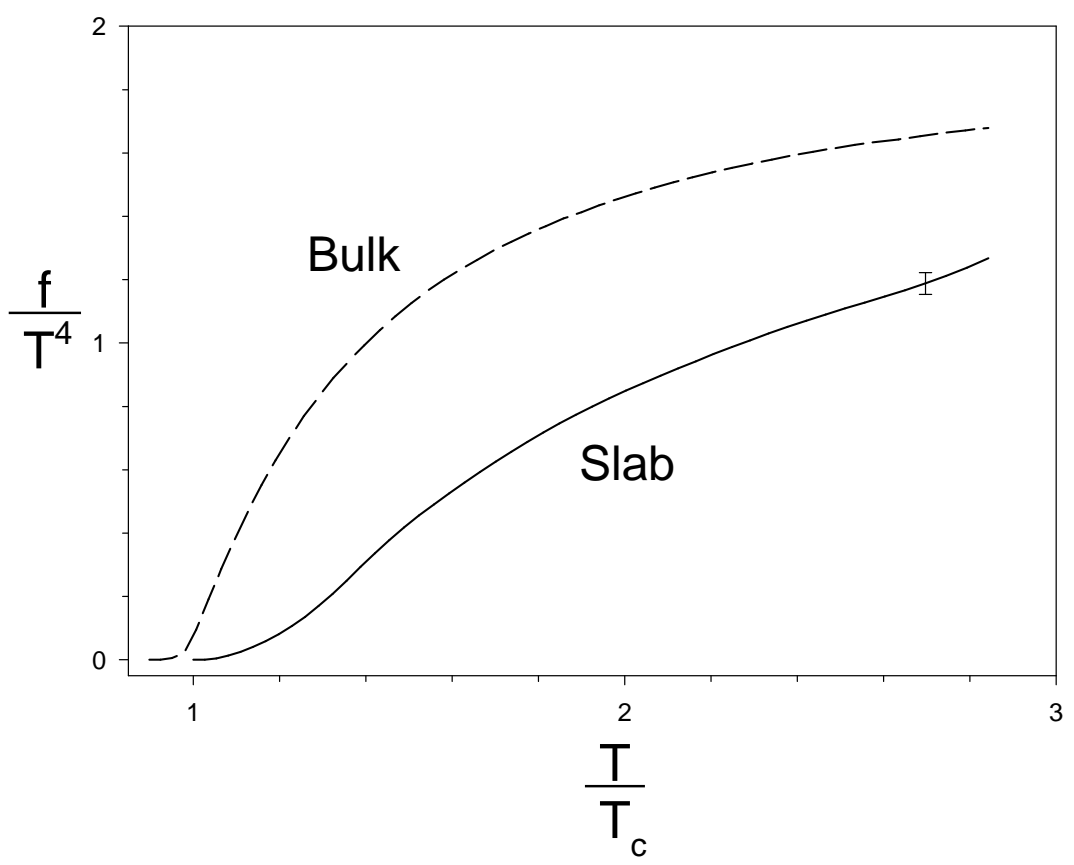


FIG. 3. Paths in the $\beta_{\text {in }}-\beta_{\text {out }}$ plane.

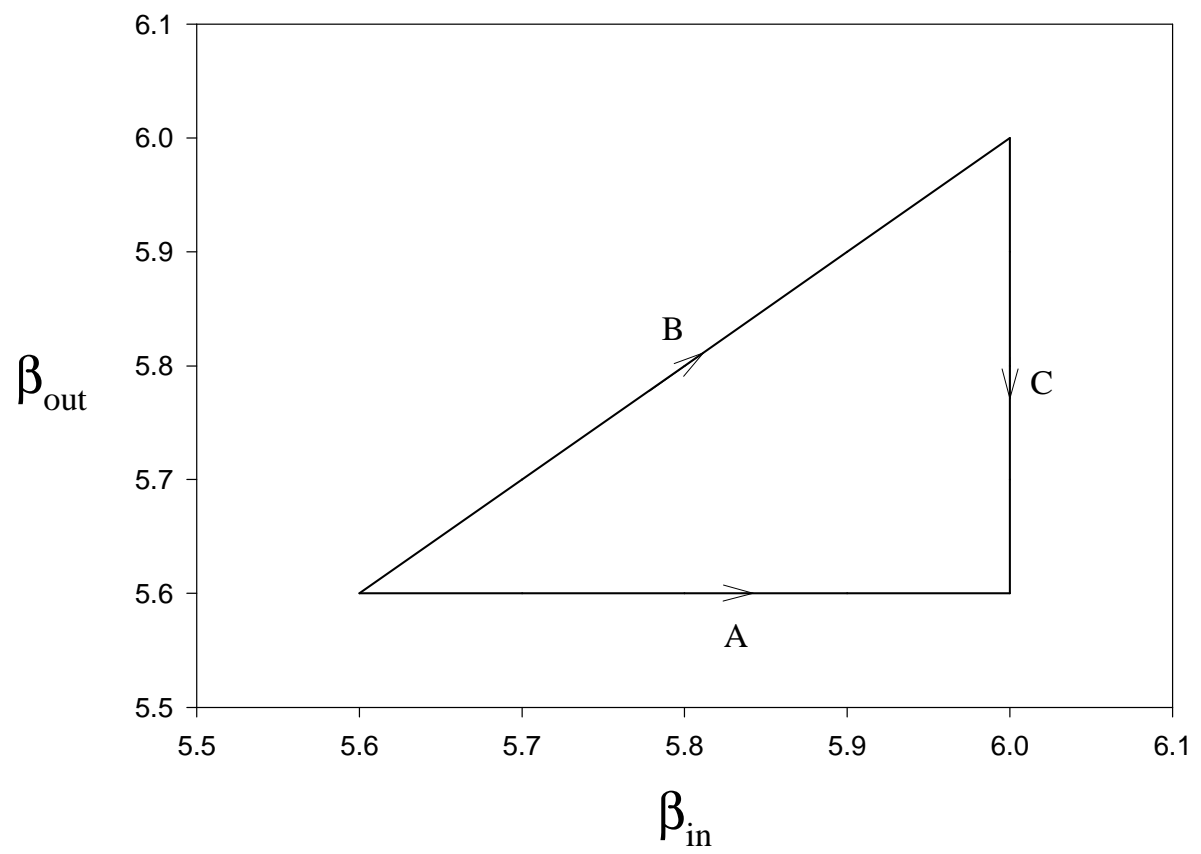


FIG. 4. Effective surface tension $\alpha / T^{3}$ versus $T / T_{c}$ for the slab geometry at $w T=3 / 2$.

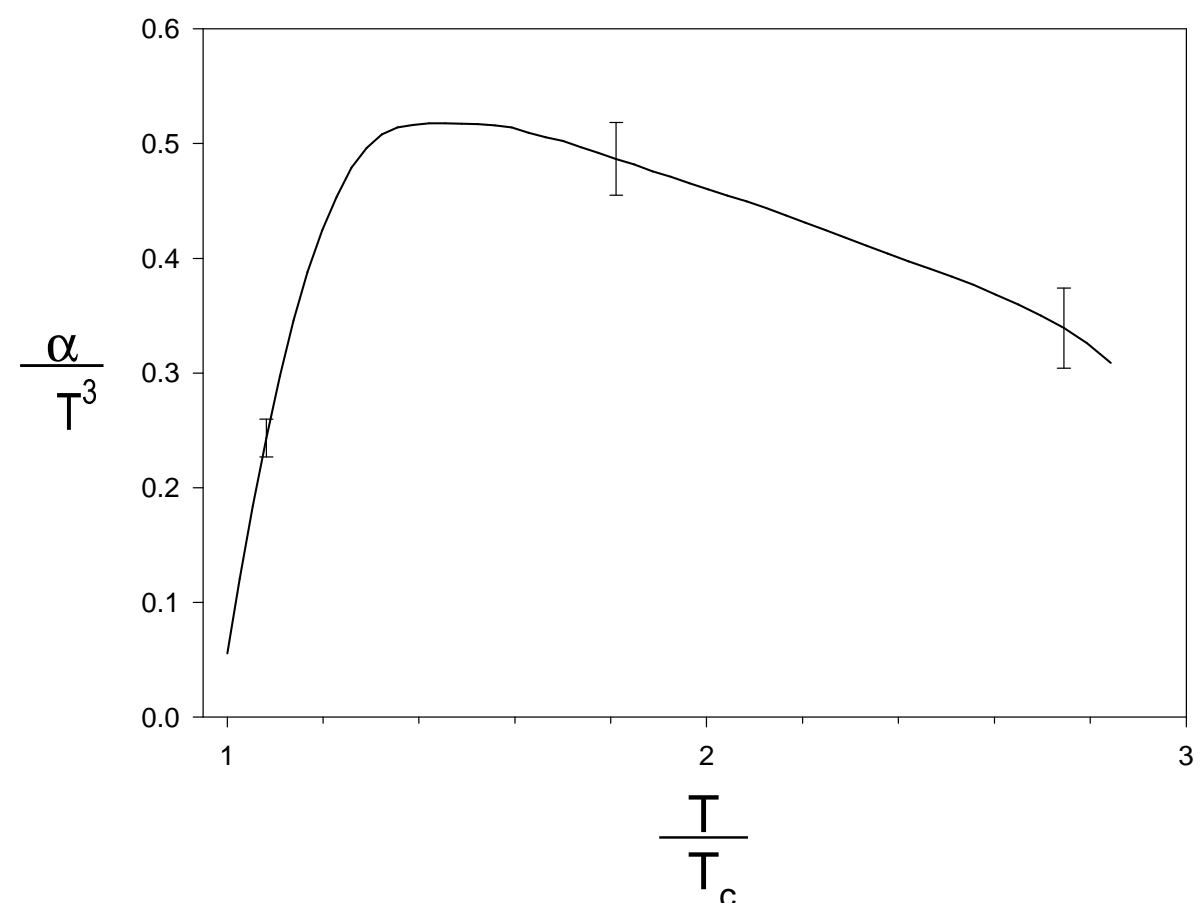


FIG. 5. Polyakov loop expectation value versus position in the $\mathrm{z}$ direction.

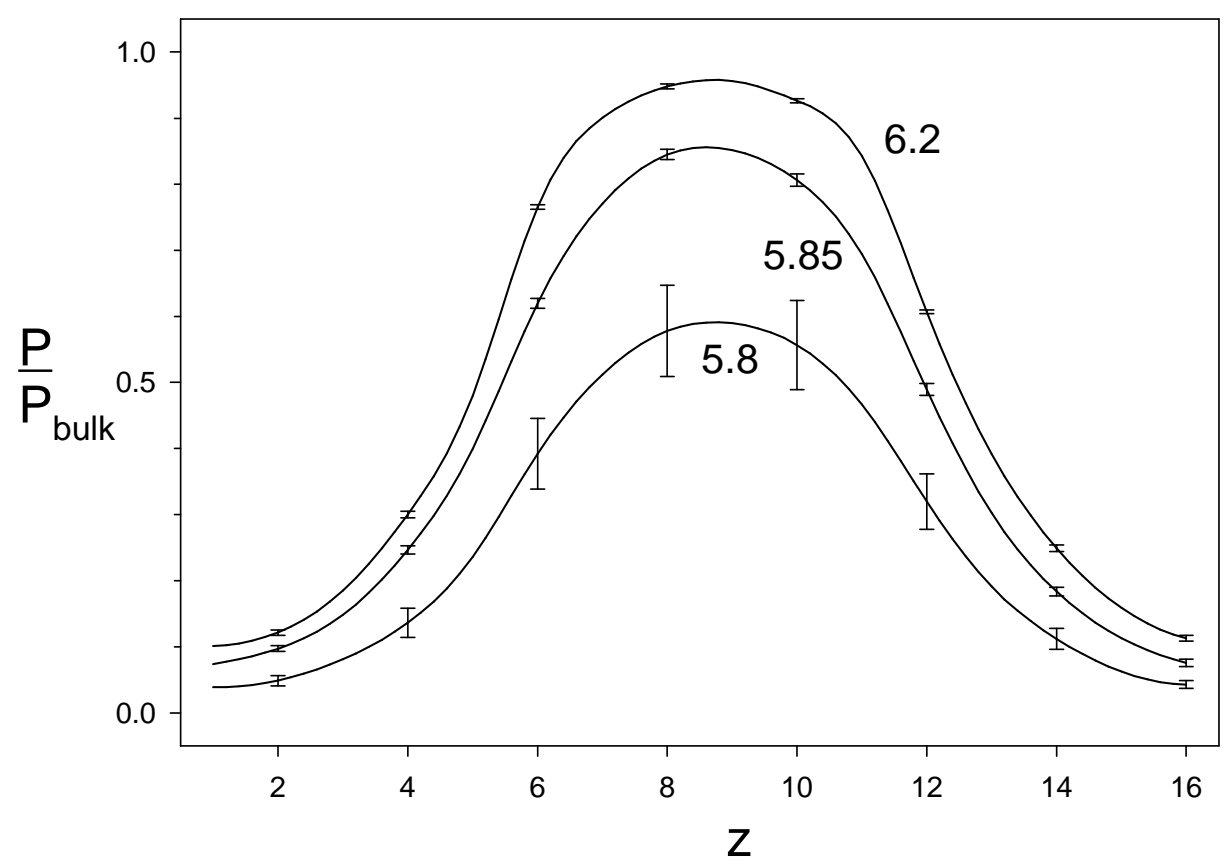

